\title{
Origin and Acceleration of Fast and Slow Solar Wind
}

\author{
Giannina Poletto \\ INAF - Arcetri Astrophysical Observatory, Largo Fermi, 5, 50125 \\ Firenze, Italy
}

\begin{abstract}
Before the advent of the Solar and Heliospheric Observatory (SOHO, launched in 1995), we had little information on how coronal plasma gets accelerated to the high speed measured in situ. The Ultraviolet Coronagraph Spectrometer (UVCS) on SOHO, acquiring UV data over the first few solar radii, a region unexplored in this spectral range, allowed us to build a profile of the outflow plasma speed vs. heliocentric distance, based on empirical constraints. Still, much has to be learnt about the behavior of solar wind in the extended corona. In the following, after briefly reviewing the general properties of the solar wind, I'll focus on controversial issues - like the identification of the sources of fast and slow wind, and the acceleration of fast vs. slow wind streams - and I'll illustrate recent contributions to the solution of these problems.
\end{abstract}

\section{Introduction}

Back in the early '70s the OSO satellites provided a wealth of data on coronal holes $(\mathrm{CH})$, quite mysterious areas, at the time, identified in 1973 as the source regions of high speed wind streams (Krieger, Timothy, \& Roelof 1973). In the same year, Noci showed, by comparing the energy budget in quiet and coronal hole regions, that also theoretical arguments lead to identify $\mathrm{CH}$ with the sites where fast wind originates. On the other hand, the continuous status of the solar wind had been ascertained about 10 years earlier from in situ observations of wind particles by the Mariner 2 spacecraft. Obviously, these results raised a question: how does the wind plasma accelerate from the negligible outflow speed at the coronal base to the high speed measured in situ?

Later observations had shown that in addition to the fast wind emanating from high latitude coronal holes there is a slow wind emanating from low latitude regions. The presence of an apparently bimodal wind poses further questions: is there a difference in the acceleration of the two components? which are the sources of the slow wind streams? also, after polar regions had been recognized to be structured, which are precisely the sites where fast wind originates?

In the present paper I'll review the observational evidence that has been acquired over the last few years and has allowed us to answer, although not always conclusively, some of the previous questions. In the first section results about plasma acceleration in coronal holes are briefly outlined and followed by a discussion on the identification, within holes, of the sources of fast wind streams. Next I'll illustrate what we know about the slow wind acceleration and, in the 
last section, I'll examine the problem of the identification of the sources of slow wind. A list of open issues concludes the paper.

\section{Acceleration of the Fast Wind}

In a landmark paper, Krieger, Timothy, \& Roelof (1973) suggested coronal holes to be the sites where fast wind originates, on the basis of the "striking" agreement between the Carrington longitude of the solar source of a recurrent high speed stream and the position of the hole. An obvious missing piece of evidence to confirm this identification, was the direct measurement of the plasma outflow speed vs. altitude profile in coronal holes: an observational test which could hardly be made, because the intensity of collisionally excited coronal lines decreases so quickly with height to vanify any attempt of a study of the Doppler shift in lines observed at increasingly higher heliocentric distances. Hence, only a few observations have been made over those and the following years to show that $\mathrm{CH}$ lines were blueshifted, relative to the rest of the disk (see, e.g., the observations in the $\lambda 625 \mathrm{Mg}$ X line by Orrall, Rottman, \& Klimchuk 1983).

However, information about the behavior of the wind plasma came from a different source: interplanetary scintillation measurements (IPS). Although these refer to density fluctuations in the wind, rather than to the speed of the wind itself, IPS observations indicated that the plasma outflow speed had reached the terminal wind speed by $\approx 10$ solar radii (Grall et al. 1996). As a consequence solar wind had to accelerate over a limited altitude interval in the corona.

The Ultraviolet Coronagraph Spectrometer aimed at providing information on the properties of plasma in this region and, in particular, on the plasma outflow speed, by taking advantage of the Doppler Dimming (DD) effect, first discussed by Hyder \& Lites (1970) and Beckers \& Chipman (1974). This effect applies to lines which form by resonant scattering of solar disk photons. In this case, in the frame of reference of the outward moving wind plasma, the exciting photons are red-shifted and the resonantly excited coronal line is Doppler dimmed because the larger the outflow speed, the fewer the scattered photons. Thus once the percentage decrease in the line intensity, with respect to a static atmosphere, is evaluated, we have a means to infer the outflow plasma speed. We refer the reader to Kohl et al. (1995) for a description of the UVCS experiment and to Withbroe, Kohl, \& Wieser (1982) and Noci, Kohl, \& Withbroe (1987) for a more thorough discussion of the Doppler dimming effect.

The two strongest lines observed by UVCS are the $\mathrm{H} L y_{\alpha}$ and the OVI 1032 and $1037 \AA$ doublet. The $\mathrm{H} L y_{\alpha}$ forms by resonant scattering of chromospheric radiation (only a negligible component of the $L y_{\alpha}$ line being due to collisional excitation) and the OVI lines have both a collisional and a radiatively excited component. Observations of these lines allow us to evaluate the proton and the OVI ion outflow speed in the corona via the DD diagnostics. Using data taken in a polar coronal hole, close to the minimum of the solar activity cycle, Cranmer et al. (1999) derived the profile of the proton and OVI ions outflow speed in the altitude interval between $\approx 1.5$ and $\approx 4$ solar radii. Figure 1 , adapted from the Cranmer et al. paper, shows results from their work. 


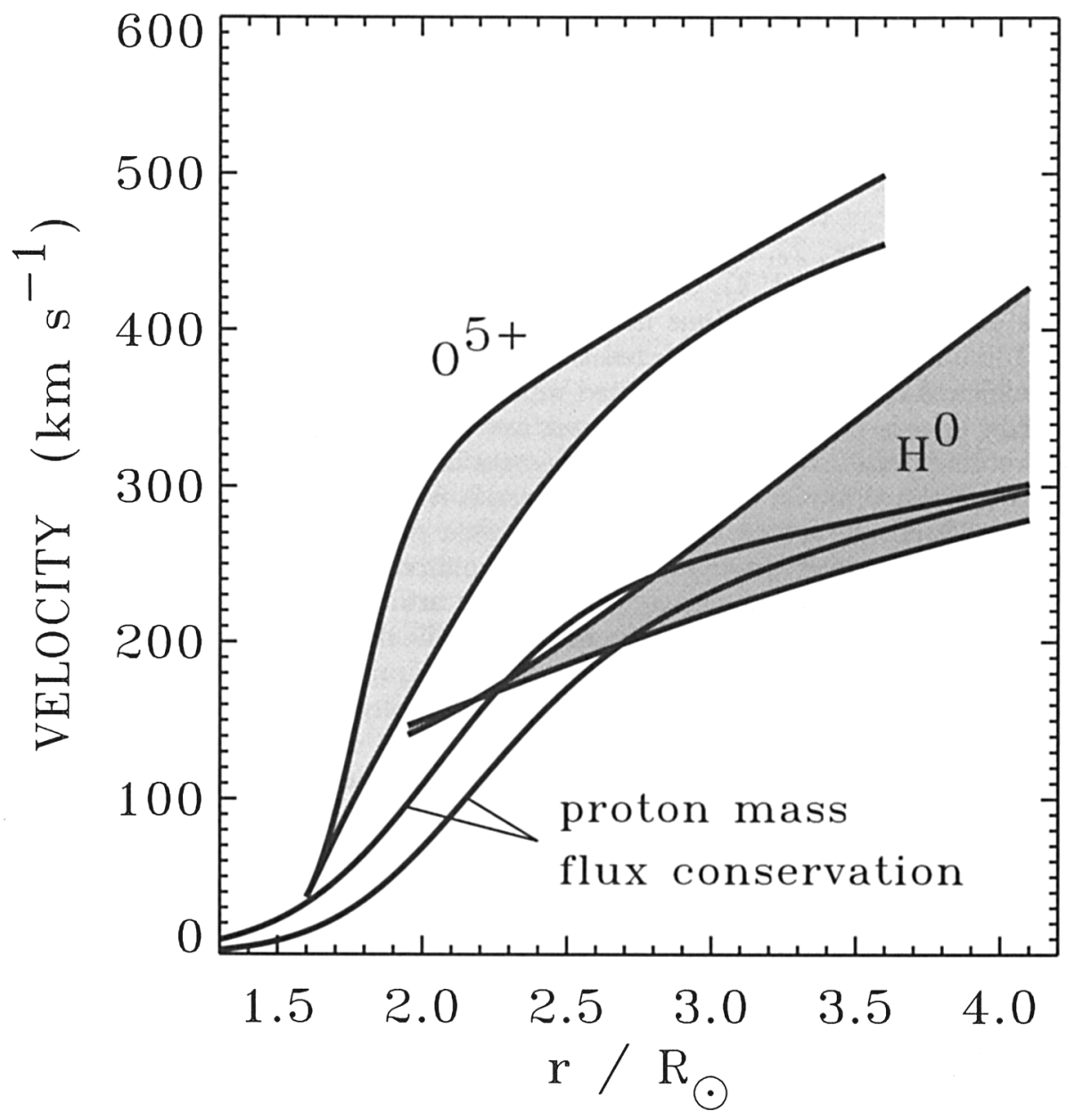

Figure 1. Gray shaded areas give the range of values for the outflow speeds of $H^{0}$ (supposedly flowing at the same speed as protons) and OVI ions which are compatible with data acquired in a polar coronal hole (Cranmer et al. 1999). The white area, which eventually superposes onto the gray $H^{0}$ area, gives outflow speed values derived from the proton mass flux conservation for different expansion factors of the coronal hole. 
Before discussing the wind profile of figure 1, I would like to point out a significant difference between speeds derived from the DD diagnostics and speeds derived from Doppler shifts. While the latter provide a direct measurement of one component of the velocity vector, outflow speeds from DD are not measured, but inferred from an analysis based on a number of parameters, often not known as precisely as desirable. We can crudely list the steps one goes through in order to build a wind speed profile, like that of Figure 1, starting from UVCS measurements at different altitudes in a polar coronal hole of the $\mathrm{H} L y_{\alpha}$ and OVI line intensities and widths. From the line widths, $T_{L O S}$, the temperature along the line of sight is easily calculated. Given this data, one should know the distribution, over the observed altitude range, of 1), coronal densities, 2), electron temperatures, $\left.T_{e}, 3\right), \mathrm{H}$ and OVI temperatures along the direction of the exciting radiation and, 4), the value of the disk intensities in $\mathrm{H} L y_{\alpha}$ and OVI lines on the day observations have been acquired. On this basis, $\mathrm{H} L y_{\alpha}$ and OVI line intensities are calculated keeping the outflow speed as a free parameter which is adjusted until predicted intensities reproduce observations. Hence, outflow speeds from the DD technique are derived from a consistency analysis where uncertainties in the input parameters impact on the derived speed value. Although the Cranmer et al. model is based on a slightly different method than outlined here, the reason why figure 1 gives a range of values compatible with observations is clear: temperatures along the direction of the exciting radiation, for instance, are not known and their values are usually assumed to lie in the range $T_{e} \lesssim T \lesssim T_{L O S}$. In spite of these difficulties, DD analyses of $\mathrm{H}$ and OVI lines in the extended corona allowed us to probe a largely unexplored region of the corona yielding severe constraints on physical parameters previously unknown.

Figure 1 shows, as expected, that wind plasma accelerates over the first few solar radii, but shows also an unexpected result: heavy ions, represented by OVI, have a higher speed and accelerate faster than protons. This result, and the large $T_{L O S}$ observed in heavy ion lines, led to a revival of theories of coronal heating based on the dissipation of ion-cyclotron resonant waves (see, e.g., Hollweg 1986; McKenzie et al. 1995; Tu \& Marsch 1997), although is not clear, yet, how these waves are generated in the extended corona (see, e.g., Cranmer \& Van Ballegooijen 2003). Discussion of these issues, however, is beyond the scope of the present paper.

A number of coronal hole speed profiles have been built, after the Cranmer et al. (1999) work. Antonucci, Dodero, \& Giordano (2000) focussed on conditions which allow protons and OVI ions to flow at the same speed; Zangrilli et al. (2002) studied the distribution of outflow speeds within a polar coronal hole with a technique which determines electron densities from the same UVCS data from which outflows are inferred. This method allowed Zangrilli et al. (2002) to avoid uncertainties introduced by temporal and spatial variations between the time when UVCS and white light data (from which densities are usually estimated) are acquired and to successfully reproduce $\mathrm{H} L y_{\alpha}$ and OVI line intensities, assuming the oxygen abundance is known. Strachan et al. (2000) used synoptic observations of the corona to reconstruct local OVI emissivities and derive the OVI ion speed $v s$. altitude $(h)$ and latitude $(\theta)$ for $\theta \lesssim 70^{\circ}$ and 
$1.75 \lesssim h \lesssim 2.75$ solar radii; Miralles et al. (2001) compared equatorial and polar outflow speeds. We will more thoroughly discuss equatorial wind in section 4 .

Most models focus on the OVI ion behavior and give an outflow speed which is consistent with the observed 1032 to 1037 line intensity ratio, but do not attempt reproducing individual intensities. While this technique (see Noci et al. 1987 for further details) avoids introducing the poorly known oxygen abundance, there is no guarantee that OVI intensities can be synthesized because the same ratio may obviously originate from quite different values of line intensities. We feel authors should pay more attention to this issue in order to strengthen the validity of their results.

\section{Sources of Fast Wind Streams}

Models described in the preceding Section used data averaged over coronal hole structures: in particular, white light, UV, soft X-ray images of polar holes revealed these to contain denser features, the plumes, which preserve their identity to large distances (see, e.g., Koutchmy 1977; DeForest et al. 1997, 2001). Theoretical works do not make definite predictions about the role of plumes as contributors to the solar wind: according to Wang (1994) and Habbal et al. (1995) plumes contribution to the high-speed wind mass flux is modest, while DelZanna (1997) and Casalbuoni et al. (1999) point out that we have an insufficient knowledge of parameters, like the temperature and the Alfvén wave flux in the two regions, to reach a definite conclusion on the plume/interplume wind relationship.

Observationally, there are indications favoring the interplume ambient as the source region of fast wind. Line widths are larger in interplumes: this has been observed at lower (see, e.g., Wilhelm et al. 1998) and at higher coronal levels (see, e.g., Noci et al. 1997) with, respectively, SUMER and UVCS, and can be interpreted in terms of a preferential energy deposition in those areas. Blue shifts are higher outside of plumes (see, e.g., Wilhelm et al. 2000); also, a DD analysis by Giordano et al. (2000) and by Patsourakos \& Vial (2000) showed higher outflows to be present in interplumes. The two latter analyses refer to only one coronal level and do not derive a profile of the outflow speed $v s$. heliocentric distance. Hence, they do not give a definitive answer to the question.

Recently Teriaca et al. (2003) used a SUMER and UVCS data file from observations acquired in a 1996 polar hole, where plumes/interplumes were detectable, to derive, via a DD analysis, the profile of the outflow plasma speed in interplumes, from the coronal base to 2 solar radii. Assuming static plumes to be immersed in this outflowing ambient, Teriaca et al. integrated along the lineof-sight over a mixed plume/interplume medium and reproduced the intensities of the OVI lines in plumes (and interplumes) over the 4 orders of magnitude variation occurring in that altitude interval. This result implies that data support a scenario where plumes, embedded in an outflowing plasma (accelerated from as low as $h \approx 1.4$ solar radii), do not contribute to the outflowing wind. We point out that an empirical estimate of the heating rate, per particle, of $\mathrm{H}$ and OVI ions, in interplumes, based on the measured kinetic temperatures and the DD outflow speeds, turns out to be in good agreement with theoretical pre- 
dictions for this quantity given in a 4-fluid model based on ion-cyclotron wave dissipation (Hu, Esser, \& Habbal 2000).

The identification of interplumes as fast wind sources, given by Teriaca et al. (2003) has been challenged by Gabriel et al. 2003 (see also this volume), who got to the conclusion that outflows are higher in plumes than in interplumes, at least in the altitude range $1.05 \lesssim h \lesssim 1.35$ solar radii they analyzed. Although the interplume outflow speed profile derived by Gabriel et al. is consistent with the Teriaca et al. profile, Gabriel et al. predict outflows to be larger by a factor $\gtrsim 2$ in plumes than in interplumes. In their model, at 1.05 solar radii, plume plasma has a speed $z 70 \mathrm{kms}^{-1}$, that keeps approximately constant beyond this level. The possibility that Teriaca et al. and Gabriel et al. analyses refer to basically different structures, with different properties, cannot be discarded, at this time, but additional work is badly needed to solve what is today a controversial issue.

\section{Slow Wind Acceleration}

The fast/slow transition in the solar wind speed originating in high/low latitudes, mentioned in the Introduction, has been clearly illustrated by measurements taken by Ulysses during its pole to pole transits (see, e.g., Phillips et al 1995; McComas et al. 2003). We may ask where the fast/slow transition observed in situ initiates: can we trace it back to the lower coronal levels or does it set in somewhere at larger heliocentric distances? In order to explore this issue, we may build an empirical model of the low latitude solar wind using the Cranmer et al. (1999) technique described in Section 2. An attempt in this direction has been made by Miralles et al. (2001) who derived the profile of the outflow speed of OVI ions in an equatorial hole, for $1.5 \lesssim h \lesssim 3$ solar radii, and conclude that OVI ions have outflow speeds about 3-4 times lower than those of the 1996 polar hole of Cranmer et al. (1999). Also, smaller blue-shifts than in polar holes have been observed at the base of equatorial holes (Buchlin \& Hassler 2000). We point out that empirical models for low latitude holes are easily subject to uncertainties arising from structures which project onto the hole areas with a contribution that can hardly be estimated.

These effects can be minimized acquiring data at higher coronal altitudes. Poletto et al. (2002) made UVCS observations at 3.5 and 4.5 solar radii, during a SOHO-Sun-Ulysses quadrature, with the aim of deriving the wind speed for protons and OVI ions at those heights and correlate coronal to in situ speed values. A quadrature configuration offers the best opportunity to compare directly plasma properties from remote coronal observations with properties of the same plasma parcel measured in situ (we refer the reader to Suess et al. 2000 for further information on the quadrature geometry and the rationale of quadrature campaigns). Because a quadrature campaign lasts typically about 2 weeks, Poletto et al. (2002) have been able to correlate variations with time of the outflow speed of coronal plasma both with the changing coronal configuration and with values measured in situ. Figure 2 from their work shows that the value of the plasma outflow speed in the corona increases from streamers to low-latitude holes and polar holes. Possibly the variability of the slow wind may, in part, be ascribed to Ulysses sampling wind originating in different regions of the Sun. 


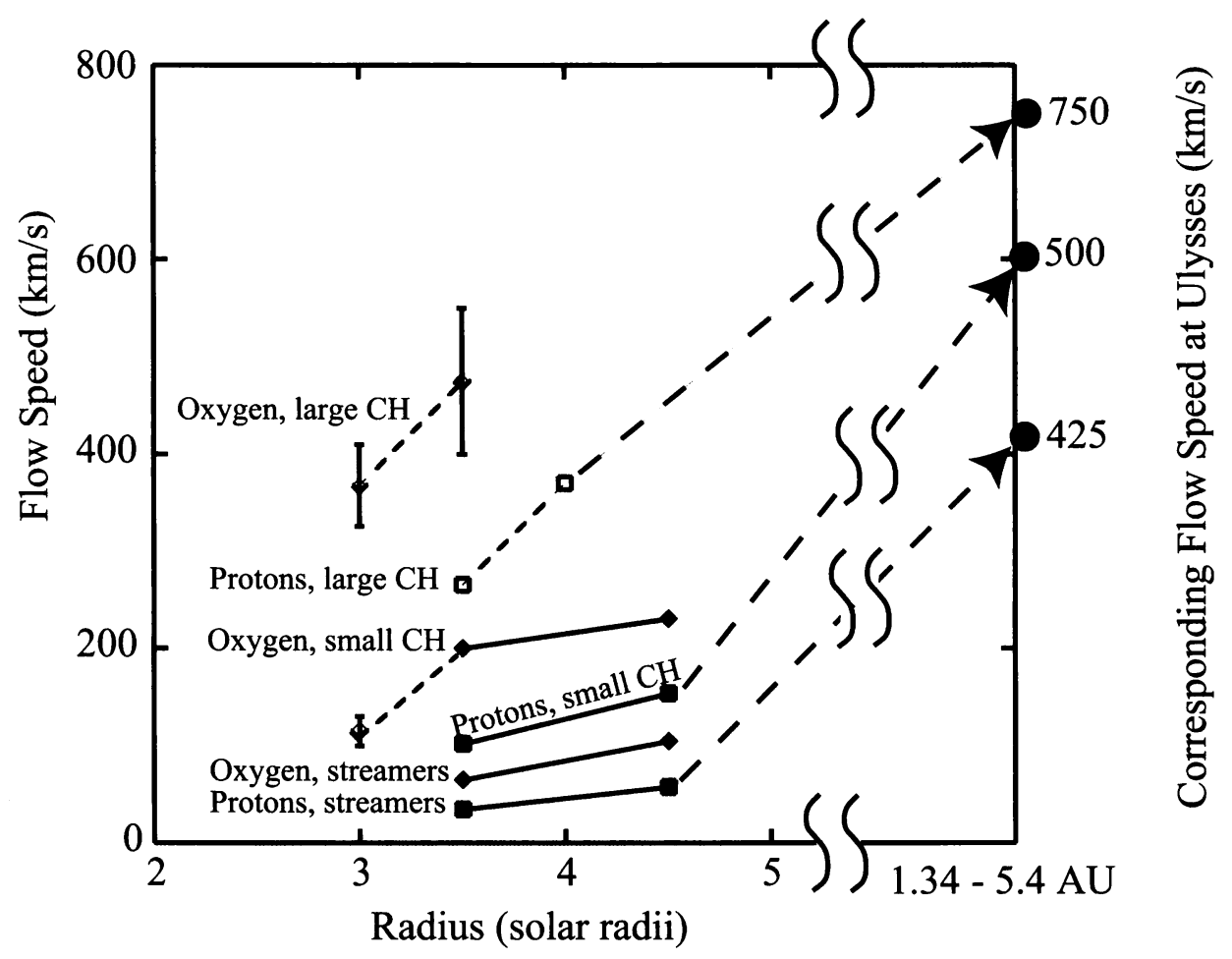

Figure 2. Solid black squares and solid black diamonds give, respectively, the proton and OVI ions outflow speed at 3.5 and 4.5 solar radii from Poletto et al. 2002; open squares are the proton flow speed from Cranmer et al. 1999 polar hole; open diamonds are the oxygen flow speed in equatorial holes from Miralles et al. 2001 and in polar holes from Cranmer et al. 1999. The terminal speed of the plasma parcels whose coronal outflows are given in the figure is shown on the right hand side axis and has been measured in situ by Ulysses. 
Figure 2 gives information on the slow wind acceleration. Wind emanating from low-latitude holes, reaches, at 3.5 solar radii, an outflow speed of $\approx 1 / 5$ of its terminal speed, as opposed to the polar wind, which, at the same level, reaches a speed of $\approx 1 / 3$ of its own terminal speed. It follows that wind from low-latitude holes accelerates over a more extended distance range than polar wind. The same kind of arguments, applied to wind emanating from streamer regions, leads to the conclusion that the distance over which plasma from streamer regions accelerates is more extended than the interval over which plasma from low-latitude holes accelerate. Apparently, the slower the outflow speed, the wider the acceleration region. We conclude that data gathered so far suggest that the slow/fast wind behavior sets in in the low corona, the outflow speed vs. altitude profile being different in fast vs. slow wind already at coronal levels.

\section{Sources of Slow Wind Streams}

The connection between wind streams and their coronal sources analyzed by Poletto et al. (2002) for slow wind quadrature data has been studied, without the help of the quadrature geometry, on a larger data set by e.g. Neugebauer et al. (1998) and Neugebauer et al. (2002), who used in situ wind data from Ulysses and Wind spacecrafts and reconstructed the coronal streams sources by extrapolating back to the Sun with different magnetic field models. They conclude that the highest speed wind comes from polar holes, while intermediate and slow wind originated either in smaller holes at low latitudes or from open regions at the boundary of holes. These findings are in agreement with predictions from theoretical models: Wang (1994), for instance, claims from model calculations that slow wind comes from small coronal holes or from boundaries of large polar holes. The inverse relationship between wind speed and expansion factors has been explained in terms of the increase of the mass flux density with increasing flux-tube divergence rate (see, e.g., Wang, Hawley, \& Sheeley 1996).

Neugebauer et al. (2002) point out that there is a further source of quasistationary wind: active regions, which produce slow and moderately fast wind. This result agrees with previous findings by Kojima et al. (1999) and Hefti et al. (2000) who identified active regions as sources of slow wind streams from in situ data. In the corona, Svestka et al. (1998) and Liewer et al. (2001) found evidence for solar wind originating in active regions, respectively, from Yohkoh and LASCO data. No direct measurement has been made, though, of plasma outflows in the corona for active region originating wind: an issue that should be further explored.

Apart from small low-latitude holes and, possibly, active regions, slow wind has been traditionally credited for being related to the highly variable streamer belt (see, e.g, Gosling 1996). However, there have been different suggestions as to where, within streamers, slow wind originates. The oxygen depletion in streamer cores, observed by UVCS (see, e.g., Kohl et al. 1997), has been interpreted by Noci et al. (1997) as being related with the origin of the slow wind. Alternative interpretations for this depletion, however, have also been proposed (see, e.g., Raymond et al. 1997). Blobs detaching from the streamer cusp and accelerating up to the typical slow wind speed have been observed with LASCO by Sheeley et al. (1997) and modeled by Einaudi et al. (2001): however, the bulk of 
slow wind is not constituted by the LASCO blobs. Streamers legs have been identified as slow wind sources on the basis both of empirical evidence, which we are going to discuss in the following, and of theoretical modeling (see, e. g., Ofman 2000). Hence, the details of the streamers/slow wind connection have not been uncontroversially established.

Raymond et al. (1997) from the similarity of the abundances in the legs of streamers at 1.5 solar radii to slow wind abundances suggested legs - as opposed to cores - to be sources of slow wind. This conclusion is not shared by Marocchi, Antonucci, \& Giordano 2001 who feel abundances in streamers legs and slow wind to be too different to justify the claim that slow wind originates primarily from streamers legs. Here it is worth reminding readers that element abundances at high coronal levels $(h \gtrsim 1.5$ solar radii) are affected by large errors due to integrating along the line of sight through possibly multiple structures at different altitudes and to a lack of information on the plasma ionization balance, which add to the usual uncertainties in line intensities and atomic rates. Also, it is difficult to ascertain how element abundances change with heliocentric distance.

An alternative way to explore the streamer/slow wind association calls for a comparison between FIP (First Ionization Potential) effects in streamers and in slow wind. It is well known that slow wind plasma has a higher FIP bias than fast wind (see, e.g., Geiss, Gloeckler, \& von Steiger 1995): approximately, we may say there is an anticorrelation between wind speed and FIP values (see, e.g., Aellig et al. 1999). This behavior suggests to look at FIP bias in the corona to find indications on the slow wind source. Recently Bemporad et al. (2003) evaluated the Fe over Oxygen ratio - which is a good proxy for the FIP bias - across streamers, at the time of a SOHO-Sun-Ulysses quadrature, and compared the coronal values with in situ values of the same ratio, measured by Ulysses/SWICS. However, Bemporad et al. were unable to get clues about the site, within streamers, where slow wind originates, because uncertainties in in situ values are large enough to cover the range of FIP variations across streamers. These authors conclude that probably absolute abundance values, more than ratios, are better suited for establishing a relationship between coronal and in situ values: the same conclusion that Uzzo et al. (2003) had reached in a study of the 1996 streamer belt.

An interesting theory on the origin of slow wind, which also entails an explanation for the FIP behavior of (fast and) slow wind, has been worked out by Fisk, Schwadron, \& Zurbuchen (1998) as a consequence of the heliospheric magnetic field scenario proposed by Fisk (1996). Because of the interplay between differential rotation and the non-radial expansion of the wind in coronal holes, magnetic flux is moved across coronal holes to be deposited at lower latitudes, where it reconnects with closed loops, which, as a consequence, disrupt and release plasma forming slow solar wind. This process is obviously intermittent and explains the variability of the slow wind. The difference in the FIP pattern of fast and slow wind has been explained (Schwadron et al. 1998) considering that fast wind flows continuously along open field lines, while elements in closed loops, supposedly heated by interactions of ions with MHD turbulence, distribute over the loops in a pattern that depends on their FIP, larger density of low-FIP elements, with respect to high-FIP elements, being found at the top 
of loops. The overall scenario outlined by these authors is fairly attractive, but it will be difficult to identify an unambiguous observational check to test its validity.

\section{Conclusions}

Before concluding this review I would like to point out that I didn't discuss factors possibly linked to the solar activity cycle. However, some of the results mentioned, for instance, in Section 4, may be valid at the phase of the solar cycle when data have been acquired, but may change with time. Data to investigate this issue are only now becoming available (see, e.g., Miralles, Cranmer, \& Kohl 2002). This issue needs to be investigated in future works.

In closing it may be useful to make a concise list of still open key questions:

- which is the role of plumes/interplumes as solar wind sources? can Doppler shifts observations in low latitude coronal holes supply evidence of the outflows predicted by some models? so far, evidence of plumes in low latitude holes is scanty (see, e.g., DelZanna \& Bromage 1999), but their analysis could provide valuable additional indications to understand the fast wind scenario. Reports on outflows in equatorial structures from Doppler shifts maps have appeared recently (Xia, Marsch, \& Curdt 2003) and need to be complemented by further evidence.

- which are the sources of slow wind? if different sources contribute to slow wind, what differentiates these contributions? can we analyse streamer boundaries and check on the slow/fast wind interface? can we improve on the present knowledge of the behavior of elemental abundances across streamers and across streamers' boundaries? is there a variation with altitude of the FIP bias?

- what causes the high variability of the slow wind? can we disentangle spatial from temporal effects? can we find evidence at coronal levels for the filamentary structure of the slow wind which is suggested by in situ observations?

- which is the spatial profile of the electron temperature in polar and equatorial holes? this issue can hardly be overestimated being crucial in fast and slow wind models as well as in evaluating element abundances. So far we have not converged on generally accepted profiles (see, e.g., David et al. 1998; Wilhelm et al. 1998).

Acknowledgments. The work of GP has been supported by MIUR, the Italian Ministry for University and Scientific Research.

\section{References}

Aellig, M. R., Hefti, S., Grünwaldt, H., Bochsler, P., Wurz, P., Ipavich, F. M., \& Hovestadt, D. 1999, J. Geophys. Res., 104, 24769

Antonucci, E., Dodero, M. A., \& Giordano, S. 2000, Solar Phys., 197, 115

Beckers, J. M., \& Chipman, E. 1974, Solar Phys., 34, 151

Bemporad, A., Poletto, G., Suess, S. T., Ko, Y.-K., Parenti, S., Riley, P., Romoli, M., \& Zurbuchen, T. Z. 2003, ApJ, 593, 1146

Buchlin, E. \& Hassler, D. M. 2000, BAAS, 32, 810

Cranmer, S. R., Kohl, J. L., Noci, G. and 28 co-authors 1999, ApJ, 511, 481 
Casalbuoni, S., DelZanna, L., Habbal, S. R., \& Velli, M. 1999, J. Geophys. Res., 104, 9947

Cranmer, S. R., \& van Ballegooijen, A.A. 2003, ApJ, 594, in press

David, C., Gabriel, A. H., Bely-Dubau, F., Fludra, A., Lemaire, P., \& Wilhelm, K. 1998, A\&A, 336, L90

DeForest, C. E., Hoeksema, J. T., Gurman, J. B., Thompson, B. J., Plunkett, S. P., Howard, R., Harrison, R.C., \& Hassler, D. M., 1997, Solar Phys. 175,393

DeForest, C. E., Plunkett, S. P, \& Andrews, M. D. 2001, ApJ, 546, 569

DelZanna, L., Hood, A. W., \& Longbottom, A. W. 1997, A\&A, 318, 963

DelZanna, L., \& Bromage, B. J. I. 1999, J. Geophys. Res., 104, 9753

Einaudi, G., Chibbaro, S., Dahlburg, R. B., \& Velli, M. 2001, ApJ, 547, 1167

Fisk, L. A. 1996, J. Geophys. Res., 101, 15547

Fisk, L. A., Schwadron, N. A., \& Zurbuchen, T. H. 1998, Space Sci. Rev., 86, 51

Gabriel, A., Bely-Dubau, F., \& Lemaire, P. 2003, ApJ, 589, 623

Giordano, S., Antonucci, E., Noci, G., Romoli, M., \& Kohl, J. L. 2000, ApJ, 531, L79

Gosling, J. T. in AIP Conf. Proc., 385, Robotic Exploration close to the Sun: Scientific Basis, ed. S. R. Habbal (New York: AIP), 17

Grall, R. R., Coles, W. A., Klingelsmith, M. T., Breen, A., Williams, P. J., Markkanen, J., \& Esser, R. 1996, Nature, 379, 429

Habbal, S. R., Esser, R., Guhathakurta, M., Sittler, E. C., \& Fisher, R. R. 1995, Geophys. Res. Lett., 22, 1465

Hefti, S., Grünwaldt, H., Bochsler, P., \& Aellig, M. R. 2000, J. Geophys. Res., 105,10527

Hollweg, J. V. 1986, J. Geophys. Res., 91, 4111

Hyder, C. L., \& Lites, B. W. 1970, Solar Phys., 14, 147

Hu, Y. Q., Esser, R., \& Habbal, S. R. 2000, J. Geophys. Res., 105, 5093

Kohl, J. L., Noci, G., Antonucci, E. \& 23 co-authors 1997, Solar Phys., 175, 613

Kojima, M., Fujiki, K., Ohmi, T., Tokamura, M., Yokobe, A., \& Hakamada, K. 1999, J. Geophys. Res., 104, 16993

Koutchmy, S. 1977, Solar Phys., 51, 399

Krieger, A. S., Timothy, A. F., \& Roelof, E. C. 1973, Solar Phys., 26, 343

Liewer, P. C., Hall, J. R., De Jong, E., \& 6 co-authors 2001, J. Geophys. Res., 106, 15903

McComas, D. J., Elliott, H. A., Schwadron, N. A., Gosling, J. T., Skoug, R. M., \& Goldstein, B. E. 2003, Geophys. Res. Lett., 30, 24

McKenzie, J. F., Banaszkievicz, M., \& Axford, W. I. 1995, A\&A, 303, L45

Miralles, M. P., Cranmer, S. R., Panasyuk, A. V., Romoli, M., \& Kohl, J. L. 2001, ApJ, 549, L257

Miralles, M. P., Cranmer, S. R., \& Kohl, J. L. 2002, in AGU Fall Meeting, abstract SH52A-0451 
Neugebauer, M., Forsyth, R. J., Galvin, A. B. \& 10 co-authors 1998, J. Geophys. Res., 103, 14587

Neugebauer, M., Liewer, P. C., Smith, E. J., Skoug, R. M., \& Zurbuchen, T. H. 2002, J. Geophys. Res., 107, SSH 13-1

Noci, G. 1973, Solar Phys., 29, 505

Noci, G., Kohl, J. L., \& Withbroe, G. L. 1987, ApJ, 315, 706

Noci, G., Kohl, J. L., Antonucci, E., \& 21 co-authors 1997, in ESA SP-404, The Corona and Solar Wind near Minimum Activity, Noordwijk: ESA Publications Division, ed. A. Wilson, 75

Noci, G., Kohl, J. L., Antonucci, E., \& 20 co-authors 1997, Adv. Sp. Res., 20, 2219

Ofman, L. 2000, Geophys. Res. Lett., 27, 2885

Orrall, F. Q., Rottman, G.J., \& Klimchuk, J. A. 1983, ApJ, 266, L65

Patsourakos, S., \& Vial, J.-C. 2000, A\&A, 341, 275

Phillips, J. L., Bame, S. J., Barnes, A., \& 8 co-authors 1995, J. Geophys. Res., 22,3301

Poletto, G., Suess, S. T., Biesecker, D. A., Esser, R., Gloeckler, G., Ko, Y.-K., \& Zurbuchen, T. H. 2002, J. Geophys. Res., 107, SSH 9-1

Raymond, J. C., Kohl, J. L., Noci, G., \& 24 co-authors 1997, Solar Phys. 175, 645

Schwadron, N. A., Fisk, L. A., \& Zurbuchen, T. H. 1999, ApJ, 521, 859

Sheeley, N. R., Wang, Y.-M., Hawley, S. H. \& 7 co-authors 1997, ApJ, 484, 472

Strachan, L., Panasyuk, A. V., Dobrzycka, D., Kohl, J. L., Noci, G., Gibson, S. E., \& Biesecker, D. A. 2000, J. Geophys. Res., 105, 2345

Suess, S. T., Poletto, G., Romoli, M., Neugebauer, M., Goldstein, B. E. \& Simnett, G. 2000, J. Geophys. Res., 105, 25033

Svestka, Z., Fárnik, F., Hudson, H. S., \& Hick, P. 1998, Solar Phys., 182, 179

Teriaca, L., Poletto, G., Romoli, M., \& Biesecker, D. A. 2003, ApJ, 588, 566

Tu, C.-Y., \& Marsch, E. 1997, Solar Phys., 171, 363

Uzzo, M., Ko, Y.-K., Raymond, J. C., Wurz, P., \& Ipavich, F. M. 2003, ApJ, 585,1062

Wang, Y.M. 1994, ApJ, 435, L153

Wang, Y.M. 1994, ApJ, 437, L67

Wang, Y.M., Hawley, S. H., \& Sheeley, N. R. 1996, Science, 271, 464

Wilhelm, K., Marsch, E., Dwivedi, B., Hassler, D. M., Lemaire, P., Gabriel, A. H., \& Huber, M. C. E. 1998, ApJ, 500, 1023

Wilhelm, K., Dammasch, I. E., Marsch, E., \& Hassler, D. M. 2000, A\&A, 353, 749

Withbroe, G. L., Kohl, J. L., Weiser, H., \& Munro, R. H. 1982, Space Sci.Rev., 33,17

Xia, L. D., Marsch, E., \& Curdt, W. 2003, A\&A, 399, L5

Zangrilli, L., Poletto, G., Nicolosi, P., Noci, G., \& Romoli, M. 2002, ApJ, 574, 477 